\title{
On the gases contained in the blood, and on respiration
}

\section{M.G. Magnus}

To cite this article: M.G. Magnus (1838) On the gases contained in the blood, and on respiration, Philosophical Magazine Series 3, 12:74, 300-302, DOI:

10.1080/14786443808649447

To link to this article: http://dx.doi.org/10.1080/14786443808649447

册 Published online: 01 Jun 2009.

Submit your article to this journal

Џll Article views: 2

Q View related articles $₫$ 
From the foregoing experiments $M$. Levol concludes :

First. That when ammonia is employed as a reagent, it may happen that the presence of copper, even in large quantity, may escape detection, if the solution also contains protoxide of iron, especially if it be supposed that this oxide is either partially or totally in the state of peroxide. It is not even requisite for this that the operation should be conducted in a close vessel, if the iron preponderates; because, when once solidified, the pellicle of oxide formed at the upper part of the solution preserves the rest from further oxidizement. Thus, in an analysis, or to discover by ammonia copper mixed with iron, it is requisite first to peroxidize the iron entirely.

Second. As no suboxide of nickel exists, it cannot occasion the same reaction; and therefore a new method of distinguishing it from copper results, when in the state of a double ammoniacal salt. This is effected by pouring an excess of solution of a protosalt of iron into the ammoniacal solution, which immediately decolorates it if copper, and not nickel, be held in solution, operating out of the contact of the air.-Annales de Chimie et de Physique, July 1837.

\section{ON THE GASES CONTAINED IN THE BLOOD, AND ON RESPIRA- TION. BY M. G. MAGNUS.}

M. Magnus remarks that it remains a question whether carbonic acid is formed in the lungs by the oxidizement of a part of the carbon in the blood by the action of the air, or whether venous blood, when it reaches the organs of respiration, contains carbonic acid ready formed, which is merely separated from it.

M. Magnus passed hydrogen gas through a solution of potash to deprive the gas of any carbonic acid which it might contain, and when it gave no precipitate with lime wate he passed it into the blood of a healthy man; the gas afterwards made to go through lime water gave a plentiful precipitate of carbonate of lime. Azotic gas similarly employed produced a like effect; and M. Magnus concludes, from these experiments, that carbonic acid exists ready formed in the blood, and consequently that it is not formed in the lungs. Carbonic acid was also separated from blood by means of the air-pump.

By using Liebig's apparatus M. Magnus found that blood contained about one-fifth of its volume of carbonic acid gas, and when it had been kept 24 hours, without emitting any bad smell, the quantity was larger. The results were confirmed by employing atmospheric air instead of hydrogen gas.

M. Magnus then ascertained the nature and proportions of all the gaseous contents of the blood. He found that 100 volumes of the arterial blood of a horse yielded

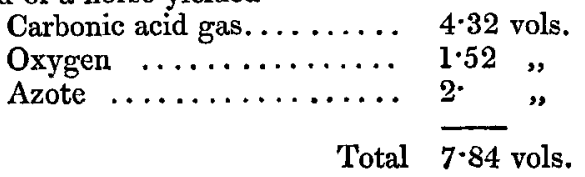

The venous blood of the same horse, drawn 4 days afterwards, gave 


$$
\begin{aligned}
& \text { Carbonic acid gas........ 4.29 vols. } \\
& \text { Oxygen ............ 1.12 , } \\
& \text { Azote } . \ldots \ldots \ldots \ldots \ldots \ldots \text {.54 ", } \\
& \text { Total } \overline{5 \cdot 95} \text { vols. }
\end{aligned}
$$

The arterial blood of the calf contains more, and the venous blood less oxygen, than that of the horse.

M. Magnus observes that these experiments, and others which we have not copied, appear to show that the gases contained in the blood of the animals, amount to about one-eighth or one-tenth of the quantity employed. He admits however that the experiments are not absolutely precise, because they were not all continued the same length of time, \&c. But he observes, that as the proportions between the oxygen and carbonic acid are invariably the same, these results may be regarded as satisfactory.

With regard to the theory of respiration all experimentalists agree as to the reciprocal proportions between the carbonic acid expired and of the oxygen absorbed; while however some of them are of opinion that those quantities are always equal, as must happen if the oxygen gas were employed merely in the formation of carbonic acid in the lungs, there are chemists whose results show that more oxygen is inspired than carbonic acid expired. Messrs. Allen and Pepys observed that this was constantly the case when the same air was repeatedly respired.

M. Magnus adds, that this fact, so inexplicable by other theories, is an immediate consequence of the hypothesis founded on the law, that a liquid holding a gas in solution parts with it when it comes in contact with another gas.

Another circumstance noticed by Messrs. Allen and Pepys is as inexplicable as the preceding, namely, that by the respiration of oxygen, or by a mixture of oxygen and hydrogen, azotic gas is constantly expired, the volume of which is proportional to the bulk of the animal; this proves that it cannot at all be attributed to the air.

It now remains to be shown that the carbonic acid extracted from the blood is in sufficient quantity to account for the whole of that which the lungs expire. The results obtained on this subject are discordant; those of Messrs. Allen aud Pepys evidently exceed what they should be ; for Berzelius has shown that, if correct, it would require six pounds and a quarter of solid nourishment in 24 hours to produce the quantity of carbon consumed.

Taking then the results obtained by Davy as a mean of those of Lavoisier, Allen and Pepys, although perhaps a little too high, we shall have 13 cubic inches as the quantity of carbonic acid gas expired by a man. If it be further admitted, that at each pulsation of the heart an ounce of blood arrives at the lungs 75 pulsations in a minute would convey five pounds of blood in the same time. This is the minimum quantity which can be admitted ; for it is very probable that five pounds of blood pass through these organs every $\mathrm{mi}$ nute : these five pounds produce 13 cubic inches. It has been already mentioned that the blood contains at least one-fifth of its volume of carbonic acid; and as a pound is equal to 25 cubic inches, each pound of blood would contain at least 5 cubic inches of carbonic 
acid. It will be observed that no circumstance opposes the proposed theory, hence the experiments prove, that the quantity of carbonic acid contained in venous blood, is more than sufficient to furnish the quantity expired.-Journal de Chimie Médicale, November 1837.

\section{ON THE LOW TEMPERATURE OF JANUARY 1838, BY MR. F. WATKINS.}

\section{To the Editors of the Philosophical Magazine and Journal. Gentlemen,}

As my own residence is so unfavourable for meteorological observations, I have had for many years, in the Blackheath road, a series of instruments under the charge of a gentleman of high scientific acquirements, and devoted to the study of the various phænomena which occur in the aërial regions. From his observations a diagram has been formed, showing the maximum and minimum thermometric curves for the month of January as compared with the mean.

The beginning of the month was mild, both curves being above the mean temperature of Greenwich, deduced from twenty years' observations. Towards the 8 th both curves descended far below the mean, and continued so till, on the 20 th, just at sun-rise, the thermometer stood at $4^{\circ}$ below 0 or zero, or $40^{\circ}$ below the mean of the period.

This low degree of temperature lasted some hours, for at 9 a.m. it was $-2^{\circ}$, at 10 a.m, $+1 \frac{1}{2}^{\circ}$ at 11 a.m. $+4^{\circ}$, and at noon only $+7^{\circ}$, after which it rose many degrees, and the wind veered from the east to south.

On the 22nd both curves ascended above the mean, and on the 23rd descended as abruptly below, accompanied by a strong easterly gale, which continued until the end of the month.

Two things may be here remarked, as being unprecedented in the annals of meteorology in this country: 1st, the thermometer below zero for some hours ; and 2ndly, followed, almost immediately after, by a variation of nearly 50 degrees. It should be noticed however from general observation that thaws commonly succeed very unusual low degrees of temperature.

My friend informs me, that before last month the lowest degree of temperature he has ever registered during thirty years, is $+4^{\circ}$, and that only once, and it was very transient.

The temperature of the month had been gradually preparing us for an extreme of cold, for on the 12 th the minimum was $12^{\circ \cdot 2}$, on the 13th $9^{\circ} \cdot 6$, and on the 15 th $6^{\circ} \cdot 5$. From the 18 th to the night of the $19 \mathrm{th}$, on which day there was only a maximum of $21^{\circ}$, the approach of an unusual degree of severity was indicated; the radiator on the snow at 6 p.m. marked $9^{\circ}$ : the evening proved cloudy with a variable temperature from $15^{\circ}$ to $12^{\circ}$. The lowest mean temperature for the month (for twenty years) falls about the 16th. Finally, the average degree of cold on our very severe night is $16^{\circ}$; therefore the thermometer on the night of the 19th departed $20^{\circ}$ lower than this, a circumstance, happily for us, of rare occurrence in this climate.

5, Charing Cross, I remain, Gentlemen, yours, \&c., Francis Watkine. 